\title{
Levels of Breast Milk PBDEs From Southern Taiwan and Their Potential Impact on Neurodevelopment
}

\author{
HOW-RAN CHAO, TSUI-CHUN TSOU, HUEI-LIN HUANG, AND GOU-PING CHANG-CHIEN
}

Department of Environmental Science and Engineering [H.-R.C.], National Pingtung University of Science and Technology, Neipu, 912 Pingtung, Taiwan; Division of Environmental Health and Occupational Medicine [T.-C.T.], National Health Research Institutes, Zhunan, Miaoli County 350, Taiwan; Institute of Behavioral Medicine [H.-L.H.], Medical College, National Cheng Kung University, 701 Tainan City, Taiwan; Department of Chemical and Materials Engineering [G.-P.C.-C.], Cheng-Shiu University, 833 Kaohsiung City, Taiwan

\begin{abstract}
In vivo studies have demonstrated that prenatal or neonatal exposure to polybrominated diphenyl ethers (PBDEs) causes developmental neurotoxicity. However, there is a lack of human data. Our hypothesis was that PBDEs would result in lower infant neurodevelopment scores. This is a post hoc analysis of previous studies. Fourteen PBDEs in 70 breast milk were analyzed using a high-resolution gas chromatograph/high-resolution mass spectrometer. Infant neurodevelopment at the age of 8-12 mo was determined using the Bayley Scales of Infants and Toddlers Development, third edition (Bayley-III). The median of $\Sigma_{14}$ PBDEs (the sum of 14 PBDE congeners) was $2.92 \mathrm{ng} / \mathrm{g}$ lipid. The $\Sigma_{14} \mathrm{PBDE}$ concentrations were not correlated with Bayley-III scores on cognitive, language, motor, social-emotional, or adaptive behavior scales. A significantly inverse association between brominated diphenyl ether (BDE)-209 and the cognitive scale was found after multivariate stepwise linear regression analyses $(B=-0.007$, adjusted $R=-0.224, p=$ $0.032)$. In contrast, the language scale was positively correlated with BDE-196 ( $B=0.096$, adjusted $R=0.315, p=0.002)$. Our results are consistent with most in vivo studies, suggesting that prenatal or postnatal exposure to BDE-209 potentially delays the neurological development.

(Pediatr Res 70: 596-600, 2011)
\end{abstract}

$\mathrm{P}$ olybrominated diphenyl ethers (PBDEs), which are only used as brominated flame retardants, are widely found in a variety of commercial and household products including foam furniture padding, plastics, electrical equipment, paints, textiles, construction materials, and vehicles (1). PBDEs are similar in structure to polychlorinated biphenyls; but in contrast to point sources of polychlorinated biphenyl contamination, PBDEs are widespread and released into the environment from more sources (2).

A marked period of rapid brain growth and development begins in humans during the third trimester of pregnancy and continues throughout the first $2 \mathrm{y}$ of life. PBDE congeners are considered to be neurotoxicants, although more work needs to be done to determine whether in utero and postnatal exposure to PBDEs has any adverse effects on human neurodevelopment, and the effects of PBDEs on human health remain unclear (3). However, recent studies have shown that human

Received February 24, 2011; accepted June 13, 2011.

Correspondence: How-Ran Chao, Ph.D., Department of Environmental Science and Engineering, National Pingtung University of Science and Technology, No. 1, Hseuh Fu Road, Neipu, Pingtung County 9127, Taiwan; e-mail: hrchao@mail.npust.edu.tw

Supported by a grant (NSC96-2628-E-020-001-MY3) from the National Science Council, Taiwan.

The authors report no conflicts of interest. exposure to PBDEs causes a reduction in women's fecundability (4), a prolongation of menstruation periods (5), an increase in serum LH in male infants (6), and disruption of thyroxine (T4), triiodothyronine (T3) and thyroid-stimulating hormones in male adults (7), and a positive association with diabetes prevalence (8).

Postnatal infant exposure to PBDEs mainly comes from breast milk and house dust (9); moreover, PBDE levels in infants and children are higher than those in adults (10). Recently, epidemiological studies of in utero exposure to PBDEs have found it to be associated with the physiological and neurological development of infants and children (6,11-16). In addition, lower birth weights were correlated with higher PBDE levels in breast milk (11) and cord blood (16), whereas prenatal PBDE exposures were related to lower cord blood levels of total T4 and free T4 (12) or T3 and free triiodothyronine (FT3) (15). Main et al. (6) found a correlation between cryptochidism in newborn boys and increased breast milk PBDE levels. However, it is still unknown how prenatal or postnatal PBDE exposure affects infant or child development, including neurodevelopment, based on the findings of current human and epidemiological studies.

Previous in vivo studies have documented that neonatal exposure to PBDEs can cause persistent neurobehavioral defects, including changes in locomotor activity, cognitive effects, spontaneous behavior, and cholinergic susceptibility (17-19). Three recent human studies showed that in utero exposure to PBDEs is linked to the neurodevelopment of young children $(13,14,20)$. Although it has been demonstrated that PBDE exposure delays the neurodevelopment of neonates and adults in animal models, few epidemiological studies have examined the correlations between infant neurodevelopment and PBDE exposure, particularly for octa-BDEs, nano-BDEs, and deca-BDE.

\section{MATERIALS AND METHODS}

Study participants. Study participants were healthy mother-infant pairs recruited from four hospitals in southern Taiwan between April 2007 and April 2010. The study protocol was reviewed and approved by the institu-

\footnotetext{
Abbreviations: Bayley-III, Bayley Scales of Infant and Toddler Development, third edition; BDE, brominated diphenyl ether; BSID-II, Bayley Scales of Infants and Toddlers Development, second edition; LODs, limits of detection; MDI, Mental Development Index; PBDEs, polybrominated diphenyl ethers; $\boldsymbol{\Sigma}_{\mathbf{3}}$ PBDEs, the sum of BDE-47, 99, and 100; $\boldsymbol{\Sigma}_{\mathbf{1 4}}$ PBDEs, the sum of 14 PBDE congeners; T4, thyroxine; T3, triiodothyronine
} 
Table 1. Descriptive statistics of study participants $(\mathrm{n}=70)$

\begin{tabular}{|c|c|c|c|c|}
\hline & Mean & Median & SD & Range \\
\hline \multicolumn{5}{|l|}{ Mothers } \\
\hline Age $(y)$ & 30.3 & 31.5 & 4.62 & $18-41$ \\
\hline Prepregnant BMI $\left(\mathrm{kg} / \mathrm{m}^{2}\right)$ & 22.5 & 21.8 & 3.73 & $15.4-34.9$ \\
\hline Parity (number)* & 1.84 & 2.00 & 0.810 & $1.00-4.00$ \\
\hline Milk lipid content (\%) & 3.51 & 3.42 & 1.05 & $1.93-7.50$ \\
\hline \multicolumn{5}{|l|}{ Infants $\dagger$} \\
\hline GA (wk) & 38.4 & 38.0 & 1.05 & $36.0-40.0$ \\
\hline Birth weight $(\mathrm{g})$ & 3140 & 3070 & 382 & $2300-4120$ \\
\hline Birth length $(\mathrm{cm})$ & 48.9 & 49.0 & 1.84 & $44.0-55.0$ \\
\hline Head circumference $(\mathrm{cm})$ & 33.5 & 33.0 & 1.24 & $31.0-36.0$ \\
\hline Chest circumference $(\mathrm{cm})$ & 32.5 & 32.5 & 1.44 & $29.5-36.0$ \\
\hline \multicolumn{5}{|l|}{ Neurodevelopment score } \\
\hline Apgar score at $1 \mathrm{~min}$ (score) & 8.68 & 9.00 & 0.577 & $6.00-10.0$ \\
\hline Apgar score at $5 \mathrm{~min}$ (score) & 8.99 & 9.00 & 0.205 & $7.00-10.0$ \\
\hline \multicolumn{5}{|l|}{ Scores of Bayley-III } \\
\hline Cognitive scale (score) & 104 & 102 & 10.7 & $85.0-130$ \\
\hline Language scale (score) & 101 & 100 & 11.1 & $77.0-124$ \\
\hline Motor scale (score) & 98.5 & 97.0 & 9.32 & $79.0-121$ \\
\hline Social-emotional scale (score) & 97.6 & 100 & 19.2 & $55.0-140$ \\
\hline Adaptive behavior scale (score) & 99.3 & 99.0 & 15.3 & $62.0-133$ \\
\hline Infant age at the time of testing (mo) & 10.9 & 11.0 & 1.05 & $8.00-12.0$ \\
\hline
\end{tabular}

$*$ The numbers of 1st, 2nd, 3rd, and 4th parity were 27, 29, 12, and 2 persons, respectively.

$\dagger$ There were 34 male infants and 36 female infants.

tional review board of the Human Ethical Committee of the Pingtung Christian Hospital, Taiwan, in 2007. The ethical standards formulated in the Helsinki Declaration of 1964 and revised in 2004 were followed. Before enrollment, all participants gave informed consent after receiving detailed explanations of the study and potential consequences. Our subjects, who were pregnant, were first interviewed by well-trained researchers at the obstetrics clinics during routine health checkups. Infants in our cohort were invited to the pediatric clinic after delivery.

The pregnant women were first selected based mainly on the following criteria: a minimum of 3 y living in southern Taiwan, an agreement to donate breast milk, not having smoked during pregnancy, a plan for breastfeeding for at least for $2 \mathrm{mo}$, and a willingness to strictly follow our protocols. More than 350 pregnant women were invited to join the program; of these, 265 agreed to answer the detailed questionnaire. Questions asked about each woman's age, prepregnant BMI, parity, socioeconomic status, smoking and dietary habits, alcohol consumption, medical history, and possible exposure to PBDEs from different sources. A total of 145 mothers were initially enrolled. Of the 145 participants, 7 women who did not offer sufficient breast milk were excluded. A total of 138 milk samples were obtained for further chemical analysis. Infants whose mothers' breast milk contained PBDEs were recruited as our cohort for follow-up evaluation in the present study. At the beginning, postcards were sent 4 mo after delivery to invite members of the cohort $(n=$ 138) to join the program. In the next step, mothers who had agreed to join the program were asked by telephone to bring their infants to the Department of Pediatrics in the Pingtung Christian Hospital for infant development evaluation. More than $70 \%$ of the infants joined the program and were reviewed by pediatricians. Twenty-six infants were excluded because they were formulatefed. Two cases did not complete the neurodevelopmental assessment. As of the cutoff date, the end of April 2010, 70 participants had been chosen to participate in the present study based on exclusive or partial breastfeeding during the first 6 mo of lactation.

Breast milk collection. Breast milk samples (120-360 mL) were collected in chemical-free glass bottles and frozen at home $\left(-4^{\circ} \mathrm{C}\right)$ within 1 mo after delivery. The breast milk samples were then transferred to our laboratory in the Department of Environmental Science and Engineering, National Pingtung University of Science and Technology, and stored at $-20^{\circ} \mathrm{C}$. Milk samples $(25 \mathrm{~mL})$ were transported to the Supermicro Mass Research and Technology Center at Cheng Shiu University in southern Taiwan for chemical analysis.

Chemical analysis of the breast milk samples. Fourteen PBDEs in breast milk-brominated diphenyl ether (BDE)-28, 47, 99, 100, 153, 154, 183, 196, $197,203,206,207,208$, and 209-were analyzed in the present study. The PBDE standards were purchased from Cambridge Isotope Laboratories (Andover, MA, USA). ${ }^{13} \mathrm{C}_{12}$-labeled standard PBDEs were sourced from Wellington Laboratories (Guelph, Canada). The highest quality sodium sulfate, alumina oxide, potassium oxalate, and silica gel were obtained from Merck (Darmstadt, Germany).
The analytical methods used to examine breast milk PBDE levels are described in our previous studies $(5,21,22)$. Briefly, milk samples with internal standards $\left({ }^{13} \mathrm{C}_{12}\right.$-labeled BDE-28, 47, 99, 153, 183, 197, 207, and 209) were extracted by sonication and then were centrifuged. The milk lipid content was determined using a gravimetric method. The extract was dissolved in $n$-hexane to be treated with concentrated sulfuric acid for the cleanup procedure by passing through a multicolumn system. The elute was concentrated to near dryness and transferred to a vial under a nitrogen stream. Breast milk PBDE levels were analyzed using a high-resolution gas chromatograph (Hewlett-Packard GC 6970; Hewlett-Packard, Palo Alto, CA) and a high-resolution mass spectrometer (Micromass Autospec Ultima, Waters, Milford, MA). Quantification was performed using internal/external standard mixtures via the isotope dilution method. Eight ${ }^{13} \mathrm{C}_{12}$-labeled PBDE internal standards were added to the breast milk before extraction to ensure recovery in the chemical analysis process. Limits of detection (LODs) were predetermined so that the signal-to-noise ratios for both ions of a specific congener would be above 3 . For measurements below the LODs, PBDE concentrations were recognized as half of the LODs.

Evaluating infant neurodevelopment. The Bayley Scales of Infants and Toddlers Development, Third Edition (Bayley-III), were used by psychologists or well-trained infant psychometrists to assess the neurodevelopment of infants between 8 and 12 mo old. The Bayley-III has three major parts: cognitive, language (receptive and expressive communication), and motor (fine and gross) (23). Parent-report questionnaires are incorporated to assess social-emotional and adaptive behavior. The assessment provides a developmental quotient, including raw scores and chronological age, and generates continuous scores for the cognitive, language, motor, social-emotional, and adaptive behavior sections. A standard score for the Bayley-III, with a mean of 100 and a SD (SD) of 15 , was derived for each scale.

Statistical analysis. PBDE concentrations are not normally distributed using the Kolmogorov-Smirnov method, but scores of the Bayley-III scale were fitted to a normal distribution. Spearman's rank correlation coefficients were initially tested to examine associations between PBDE levels and scores on the Bayley-III scale. To examine multivariate stepwise linear regression models of the Bayley-III, associations between the five scales of Bayley-III and PBDEs were determined independent of maternal age, prepregnant BMI, $\mathrm{GA}$, and infant age at the time of testing. Analyses were carried out using the Statistical Package for Social Science (SPSS) version 12.0 (SPSS Inc., Chicago, IL).

\section{RESULTS}

Descriptive statistics of participants' characteristics and breast milk PBDE levels. In Table 1, the mean and SD of maternal age and prepregnant BMI were $30.5 \pm 4.62 \mathrm{y}$ and 
Table 2. Descriptive statistics of breast milk PBDE levels collected from our subjects $(\mathrm{n}=70)$

\begin{tabular}{|c|c|c|c|c|c|c|c|c|}
\hline & $\mathrm{N}<\mathrm{LOD}$ & Mean & SD & Range & 25th Percentile & 50th Percentile & 75th Percentile & 95th Percentile \\
\hline BDE-28 (ng/g lipid) (3Br) & $0 / 70^{*}$ & 0.142 & 0.526 & $0.0186-4.41$ & 0.0382 & 0.0574 & 0.0898 & 0.301 \\
\hline BDE-47 (ng/g lipid) (4Br) & $0 / 70$ & 1.90 & 9.71 & $0.207-80.4$ & 0.358 & 0.475 & 0.643 & 1.56 \\
\hline BDE-99 (ng/g lipid) (5Br) & $0 / 70$ & 0.460 & 2.34 & $0.0418-19.7$ & 0.107 & 0.144 & 0.188 & 0.660 \\
\hline BDE-100 (ng/g lipid) (5Br) & $0 / 70$ & 0.458 & 1.53 & $0.0684-10.4$ & 0.127 & 0.172 & 0.275 & 0.522 \\
\hline BDE-153 (ng/g lipid) (6Br) & $0 / 70$ & 1.11 & 1.36 & $0.361-10.2$ & 0.630 & 0.835 & 1.09 & 2.32 \\
\hline BDE-154 (ng/g lipid) (6Br) & $0 / 70$ & 0.118 & 0.163 & $0.0171-0.900$ & 0.0415 & 0.0637 & 0.131 & 0.478 \\
\hline BDE-183 (ng/g lipid) (7Br) & $1 / 70$ & 0.236 & 0.717 & $<$ LOD-6.00 & 0.0763 & 0.110 & 0.174 & 0.576 \\
\hline BDE-196 (ng/g lipid) (8Br) & $1 / 70$ & 0.0410 & 0.0460 & $<$ LOD-0.281 & 0.0191 & 0.0267 & 0.0438 & 0.143 \\
\hline BDE-197 (ng/g lipid) (8Br) & $0 / 70$ & 0.300 & 0.338 & $0.0656-2.20$ & 0.0133 & 0.199 & 0.361 & 1.08 \\
\hline BDE-203 (ng/g lipid) (8Br) & $1 / 70$ & 0.0771 & 0.0849 & $<$ LOD-0.593 & 0.0336 & 0.0497 & 0.0985 & 0.269 \\
\hline BDE-206 (ng/g lipid) (9Br) & $0 / 70$ & 0.0623 & 0.0609 & $0.0113-0.294$ & 0.0222 & 0.0415 & 0.833 & 0.227 \\
\hline BDE-207 (ng/g lipid) (9Br) & $0 / 70$ & 0.197 & 0.223 & $0.0203-1.05$ & 0.0731 & 0.116 & 0.223 & 0.810 \\
\hline BDE-208 (ng/g lipid) (9Br) & $2 / 70$ & 0.0689 & 0.0669 & $<$ LOD-0.309 & 0.0381 & 0.0244 & 0.0440 & 0.236 \\
\hline BDE-209 (ng/g lipid) (10Br) & $1 / 70$ & 0.468 & 0.403 & $<$ LOD-1.70 & 0.221 & 0.295 & 0.565 & 1.58 \\
\hline$\Sigma_{14} \mathrm{PBDEs}$ (ng/g lipid) & $0 / 70$ & 5.64 & 14.5 & $1.44-118$ & 2.15 & 2.92 & 4.04 & 13.3 \\
\hline
\end{tabular}

* The ratio of number/number meant that the number lower than LODs was divided by the total number.

Table 3. Correlations between breast milk PBDE levels and scores of Bayley-III scale were examined by Spearman's rank correlation tests

\begin{tabular}{|c|c|c|c|c|c|}
\hline \multirow[b]{2}{*}{ PBDE congeners } & \multicolumn{5}{|c|}{ Bayley-III scale for infant neurodevelopment } \\
\hline & Cognitive & Language & Motor & Social-emotional & Adaptive behavior \\
\hline BDE-28 & $0.079(0.515)^{*}$ & $0.020(0.871)$ & $0.136(0.260)$ & $-0.145(0.232)$ & $0.026(0.832)$ \\
\hline BDE-47 & $0.065(0.591)$ & $0.129(0.286)$ & $0.043(0.723)$ & $-0.172(0.154)$ & $-0.056(0.644)$ \\
\hline BDE-99 & $-0.107(0.376)$ & $0.127(0.295)$ & $-0.176(0.146)$ & $-0.180(0.135)$ & $-0.119(0.325)$ \\
\hline BDE-100 & $0.152(0.209)$ & $0.237(0.048 \dagger)$ & $0.137(0.257)$ & $-0.085(0.486)$ & $0.051(0.676)$ \\
\hline BDE-153 & $0.021(0.861)$ & $0.060(0.623)$ & $0.158(0.191)$ & $-0.131(0.280)$ & $0.009(0.939)$ \\
\hline BDE-154 & $0.102(0.401)$ & $0.179(0.137)$ & $0.093(0.442)$ & $-0.075(0.535)$ & $0.044(0.715)$ \\
\hline BDE-183 & $0.042(0.731)$ & $0.163(0.177)$ & $-0.003(0.978)$ & $0.103(0.349)$ & $0.038(0.754)$ \\
\hline BDE-196 & $-0.059(0.630)$ & $0.179(0.138)$ & $0.174(0.149)$ & $0.079(0.515)$ & $0.019(0.879)$ \\
\hline BDE-197 & $0.035(0.772)$ & $0.162(0.180)$ & $0.132(0.278)$ & $0.120(0.323)$ & $0.011(0.929)$ \\
\hline BDE-203 & $-0.105(387)$ & $0.112(0.356)$ & $0.181(0.134)$ & $0.154(0.202)$ & $0.066(0.586)$ \\
\hline BDE-206 & $-0.219(0.068)$ & $-0.013(0.915)$ & $0.177(0.142)$ & $-0.051(0.674)$ & $-0.011(0.930)$ \\
\hline BDE-207 & $-0.154(0.203)$ & $0.059(0.626)$ & $0.085(0.485)$ & $-0.009(0.944)$ & $-0.042(0.731)$ \\
\hline BDE-208 & $-0.169(0.161)$ & $0.042(0.731)$ & $0.158(0.192)$ & $0.002(0.986)$ & $0.001(0.991)$ \\
\hline BDE-209 & $-0.267(0.026 \dagger)$ & $0.084(0.498)$ & $0.146(0.228)$ & $-0.142(0.241)$ & $-0.006(0.959)$ \\
\hline$\Sigma_{14}$ PBDEs & $-0.020(0.871)$ & $0.142(0.240)$ & $0.159(0.189)$ & $-0.064(0.599)$ & $-0.020(0.870)$ \\
\hline
\end{tabular}

* Spearman's rho correlation coefficient ( $p$ value).

$\dagger p<0.05$.

Table 4. Significant associations of infant neurodevelopment with breast milk PBDE levels using multiple stepwise linear regression tests $*$

\begin{tabular}{ccccc}
\hline \multirow{2}{*}{$\begin{array}{c}\text { Dependence } \\
\text { neurodevelopment }\end{array}$} & \multicolumn{4}{c}{ Independence } \\
\cline { 2 - 5 } & PBDEs & $B$ & Adjusted $R$ & $p$ \\
\hline Cognitive & BDE-209 & -0.007 & -0.224 & 0.032 \\
Language & BDE-196 & 0.096 & 0.315 & 0.002 \\
\hline
\end{tabular}

* The significant levels were adjusted for maternal age and prepregnant BMI, infant's gender, GA, and infant age at the time of testing.

$22.5 \pm 3.73 \mathrm{~kg} / \mathrm{m}^{2}$, respectively. Thirty-four infants were male $(34 / 70,49.8 \%)$. The mean GA was 38.4 wk, with an SD of $1.05 \mathrm{wk}$. The age at which infants were tested using the Bayley-III ranged from 8 to 12 mo, with the mean being 10.9 mo. Scores on the Bayley-III were $104 \pm 10.7$ on the cognitive scale, $101 \pm 11.1$ on the language scale, $98.5 \pm 9.32$ on the motor scale, $97.6 \pm 19.2$ on the social-emotional scale, and $99.3 \pm 15.3$ on the adaptive behavior scale. The mean and median of $\Sigma_{14}$ PBDEs (the sum of 14 PBDE congeners) were 5.64 and $2.92 \mathrm{ng} / \mathrm{g}$ lipid, respectively, as shown in Table 2.
Levels of PBDE congeners and $\Sigma_{14}$ PBDEs did not fall into an approximately normal distribution or log-normal distribution. Extremely high levels of $\Sigma_{14}$ PBDEs (113 ng/g lipid) and BDE-47 (80.4 ng/g lipid) were found in the present study; and the predominant PBDEs (BDE-47, 153, and 209) accounted for $61.7 \%$ of the total.

Correlations between PBDE exposures and neurodevelopment. Table 3 shows a negative association between BDE-209 and the cognitive scale using the Spearman's rank correlation test $(r=-0.267, p=0.026)$. In contrast, BDE-100 was positively associated with the language scale $(r=0.237, p=0.048)$. No significant associations between breast milk levels of certain PBDEs and $\Sigma_{14}$ PBDEs and the motor, social-emotional, and adaptive behavior scales on the Bayley-III test were found. To examine multiple stepwise linear regression models for the Bayley-III, a doubling of BDE-209 was associated with cognitive scores 0.007 points lower after adjusting for maternal age and prepregnant BMI, infant gender, GA, and infant age at the time of testing ( $r=$ $-0.224, p=0.032$; Table 4), whereas a doubling of 
BDE-196 was correlated with the increase of 0.096 points on language scores $(r=0.315, p=0.002)$.

\section{DISCUSSION}

To adjust for confounders, breast milk BDE-209 was negatively associated with cognitive development, but BDE-196 was positively correlated with language development in the present study. Despite Taiwan having phased out the import of penta- and octa-BDEs in 2004, the widespread use of decaBDE products that consist of BDE-209, nano-BDEs, and a few octa-BDEs (i.e. BDE-196) has resulted in accumulation in environmental matrices and biota including human bodies. This is the first report to consider the impact of highbrominated PBDEs from octa $(8 \mathrm{Br})$ to deca $(10 \mathrm{Br})$ on infant neurodevelopment. Currently, only three environmental epidemiological studies have addressed associations between infants', toddlers', or children's developmental neurotoxicity and in utero or postnatal PBDE exposure $(13,14,20)$.

Using the Bayley Scales of Infants and Development Version II (BSID-II), a recent report on prenatal exposure to PBDEs and neurodevelopment examined whether cord blood BDE-47, 99, 100, 153, 154, and 183 affected neurodevelopment at 1-4 and 6 y (14). The authors indicated that associations were inversely significant for the 12-mo Psychomotor Development Index (PDI; BDE-47), 24-mo Mental Development Index (MDI; BDE-47, 99, and 100), 36-mo MDI (BDE100), 48-mo full-scale and verbal IQ (BDE-47, 99, and 100), and 48-mo and 72-mo performance IQ (BDE-100) (14). Unlike Herbstman et al., we found that only breast milk BDE100 had a statistically positive correlation with the language scale among PBDEs from tetra $(4 \mathrm{Br})$ to hepta $(7 \mathrm{Br})$. The scale scores from Bayley-III and BSID-II are not comparable due to structural difference between these two scales, and direct comparisons with previous BSID-II studies are problematic (24). Our results cannot be compared with the findings of Herbstman et al. (14). Several factors, however, may account for the observed incomparability, including major differences in evaluation tools (Bayley-III versus BSID-II), exposure levels (the median $\Sigma_{6}$ PBDE-comprising BDE-47, 99, 100, 153,154 , and 183 - in our population was approximately 10 times lower), PBDE congeners (detecting PBDEs from octa to deca, except for tetra to hepta), sample size (70 in our study versus 98-118 in Herbstman et al.), and exposure pattern (BDE-153 was prominent in our study, in contrast to BDE-47 in Herbstman et al.).

Another recent report examined the relationship between the neurodevelopment of children 5 to 6 y old and those with prenatal exposure to PBDEs (13). Roze et al. indicated that prenatal PBDE exposure was probably related to several adverse and beneficial health effects. For instance, negative associations between PBDE exposure and children's neurodevelopment were shown for fine manipulative abilities (BDE154), verbal memory (BDE-153), and sustained attention (BDE47, 99, and 100), and positive correlations were found for total behavioral outcome (BDE-99 and 100) and internalizing behavior (BDE-47, 99, and 100). Although similar PBDEs exposure levels ( $\Sigma_{5}$ PBDEs including BDE-47, 99, 100, 153, and154:
$1.69 \mathrm{ng} / \mathrm{g}$ lipid in our study versus $3.4 \mathrm{ng} / \mathrm{g}$ lipid in Roze et al.), PBDE patterns (BDE-153 was the predominant), and sample sizes (70 in our study versus 62 in Roze et al.) were examined in both our study and that of Roze et al., our findings were not linked to those of Roze et al., mainly because of differences in the evaluation tools used (Bayley-III in our study versus the Wechsler Preschool and Primary Scale of Intelligence, Revised Edition (WPPSI-R) in Roze et al.) and the ages of the study subjects (8-12 mo old in our study versus 5-6 y old in Roze et al.).

Currently, a new study is examining associations between prenatal ( $n=88$ in cord blood) and postnatal PBDE $(n=244$ in serum from 4-y-old children) exposure and neurodevelopment and neurobehavioral development in 4-y-old children (20). Gascon et al. (20) reported that prenatal and postnatal exposure to PBDEs (BDE-47, 99, and 100) and $\Sigma_{3}$ PBDEs (the sum of BDE-47, 99, and 100) was not significantly correlated with cognitive and motor values. In the present study, $\Sigma_{3}$ PBDEs and BDE-47, 99, and 100 in breast milk was not statistically associated with cognition and motor scores, but PBDE exposure levels (median $\Sigma_{3}$ PBDEs: $0.795 \mathrm{ng} / \mathrm{g}$ lipid in our study versus $2.86 \mathrm{ng} / \mathrm{g}$ lipid in Gascon et al.), sample size (70 in our study versus 88-244 in Gascon et al.), evaluation tools (Bayley-III in our study versus McCarthy scales of Children's Abilities in Gascon et al.), and subject age (8-12 mo in our study versus 4 y olds in Gascon et al.) were different in Gascon's report. There is no information on the impact of PBDEs from octa to deca exposure on neurodevelopment or neurobehavioral development of infants or children in these three recent reports $(13,14,20)$.

Infants nursed with breast milk containing high levels of BDE-209 might have delayed cognitive development at $1 \mathrm{y}$ of age in this study, but BDE-196 might help language development (Table 4). Sensorimotor development, object relatedness, exploration and manipulation, memory, concept formation, and simple problem solving abilities are assessed using the cognitive scale, whereas the language scale includes both expressive (babbling, gesturing, and utterances) and receptive (verbal comprehension and vocabulary) communication tests $(23,24)$. Few in vivo studies have examined whether BDE-196 exposure affects neurodevelopment in neonates and adults. It is still unknown why BDE-196 had a beneficial impact on infant language development in the present study. A possible mechanism is that PBDE exposure affects neonatal neurodevelopment by interfering with the secretion of thyroid hormones. A Dutch cohort study indicated increased cord blood $\mathrm{T} 3$ in relation to prenatal exposure to BDE-47, 99, and 100, while these three PBDEs were also correlated with increases in total behavioral outcome or internalizing behavior (13). Despite the fact that our results show a positive correlation between BDE-196 and the language scale, future studies should further investigate the effects of PBDE exposure on infant neurodevelopment. In addition, neonatal BDE-209 exposure has been demonstrated to have neurotoxic effects in most in vivo studies. Neonatal exposure to BDE-209 has been found to have developmental neurotoxicity, including hyperactivity; learning and memory defects; a reduction in habituation; a decrease in hippocampal nicotinic receptors; changes 
in spontaneous and cognitive behaviors; a change in locomotor activity; changes in protein of BDNF (brain-derived neurotrophic factor), CaMKII (calcium/calmodulin-dependent protein kinase II), and GAP-43 (growth associated protein$43)$; and delays in sensorimotor development $(18,19,25,26)$. To our knowledge, epidemiological studies have yet to examine associations between infant exposure to BDE-209 and infant neurodevelopment. Extremely high levels of BDE-209 in breast milk, cord blood, and serum were found in e-waste recycling sites compared with those in the general population (27). Water supply is also a potential concern for PBDE exposure if the infants are formula-fed. In addition to PBDEs, lead $(\mathrm{Pb})$, which is also found in drinking water (28), is a neurotoxicant for infant neurodevelopment, particularly for formula-fed infants. Although BDE-209 has a short half-life compared with most PBDE congeners in the environment, it may degrade to less-brominated PBDE compounds and its toxicity remains to be determined (29). It is worth noting that BDE-209 is commonly used as a brominated fire retardant in electronic equipment and that it constitutes approximately $80 \%$ of the world market demand for PBDEs. Evaluation of health effects for human exposure to BDE-209, particularly for infants or toddlers in hotspot areas (i.e. e-waste recycling sites), is thus needed in the future.

\section{CONCLUSIONS}

High-brominated PBDE congeners with background-level exposure might affect infant neurodevelopment. The results of this study show that infants exposed to BDE-209 probably experiences developmental delays in cognition, whereas BDE196 seems to have enhanced language development. However, these findings are not conclusive because of the small size of our sample, which was only 70 infants. Consequently, larger and longitudinal epidemiological studies to examine whether PBDE exposure causes developmental neurotoxicity in infants and children are required.

Acknowledgments. We thank Ms. Eileen Ouyang for his assistance in inviting participants. We would also like to acknowledge the psychology technologists from Prof. Huang's team for their technical support in the evaluation of Bayley-III.

\section{REFERENCES}

1. de Wit CA 2002 An overview of brominated flame retardants in the environment. Chemosphere 46:583-624

2. Siddiqi MA, Laessig RH, Reed KD 2003 Polybrominated diphenyl ethers (PBDEs): new pollutants - old diseases. Clin Med Res 1:281-290

3. Birnbaum LS, Cohen Hubal EA 2006 Polybrominated diphenyl ethers: a case study for using biomonitoring data to address risk assessment questions. Environ Health Perspect 114:1770-1775

4. Harley KG, Marks AR, Chevrier J, Bradman A, Sjödin A, Eskenazi B 2010 PBDE concentrations in women's serum and fecundability. Environ Health Perspect 118:699-704

5. Chao HR, Shy CG, Chen FA, Wang SL, Chen SC, Koh TW, Chang-Chien GP, Tsou TC 2010 Impact of non-occupational exposure to polybrominated diphenyl ethers on menstruation characteristics of reproductive-age females. Environ Int 36:728-735
6. Main KM, Kiviranta H, Virtanen HE, Sundqvist E, Tuomisto JT, Tuomisto J, Vartiainen T, Skakkebaek NE, Toppari J 2007 Flame retardants in placenta and breast milk and cryptorchidism in newborn boys. Environ Health Perspect 115:1519-1526

7. Turyk ME, Persky VW, Imm P, Knobeloch L, Chatterton R, Anderson HA 2008 Hormone disruption by PBDEs in adult male sport fish consumers. Environ Health Perspect 116:1635-1641

8. Lim JS, Lee DH, Jacobs DR Jr 2008 Association of brominated flame retardants with diabetes and metabolic syndrome in the U.S. population, 2003-2004. Diabetes Care 31:1802-1807

9. Wu N, Herrmann T, Paepke O, Tickner J, Hale R, Harvey LE, La Guardia M, McClean MD, Webster TF 2007 Human exposure to PBDEs: associations of PBDE body burdens with food consumption and house dust concentrations. Environ Sci Technol 41:1584-1589

10. Toms LM, Sjödin A, Harden F, Hobson P, Jones R, Edenfield E, Mueller JF 2009 Serum polybrominated diphenyl ether (PBDE) levels are higher in children (2-5 years of age) than in infants and adults. Environ Health Perspect 117:1461-1465

11. Chao HR, Wang SL, Lee WJ, Wang YF, Papke O 2007 Levels of polybrominated diphenyl ethers (PBDEs) in breast milk from central Taiwan and their relation to infant birth outcome and maternal menstruation effects. Environ Int 33:239-245

12. Herbstman JB, Sjödin A, Apelberg BJ, Witter FR, Halden RU, Patterson DG, Panny SR, Needham LL, Goldman LR 2008 Birth delivery mode modifies the associations between prenatal polychlorinated biphenyl (PCB) and polybrominated diphenyl ether (PBDE) and neonatal thyroid hormone levels. Environ Health Perspect 116:1376-1382

13. Roze E, Meijer L, Bakker A, Van Braeckel KN, Sauer PJ, Bos AF 2009 Prenatal exposure to organohalogens, including brominated flame retardants, influences motor, cognitive, and behavioral performance at school age. Environ Health Perspect 117:1953-1958

14. Herbstman JB, Sjödin A, Kurzon M, Lederman SA, Jones RS, Rauh V, Needham LL, Tang D, Niedzwiecki M, Wang RY, Perera F 2010 Prenatal exposure to PBDEs and neurodevelopment. Environ Health Perspect 118:712-719

15. Lin SM, Chen FA, Huang YF, Hsing LL, Chen LL, Wu LS, Liu TS, Chang-Chien GP, Chen KC, Chao HR 2011 Negative associations between PBDE levels and thyroid hormones in cord blood. Int J Hyg Environ Health 214:115-120

16. Wu K, Xu X, Liu J, Guo Y, Li Y, Huo X 2010 Polybrominated diphenyl ethers in umbilical cord blood and relevant factors in neonates from Guiyu, China. Environ Sci Technol 44:813-819

17. Viberg H, Johansson N, Fredriksson A, Eriksson J, Marsh G, Eriksson P 2006 Neonatal exposure to higher brominated diphenyl ethers, hepta-, octa-, or nonabromodiphenyl ether, impairs spontaneous behavior and learning and memory functions of adult mice. Toxicol Sci 92:211-218

18. Viberg H, Fredriksson A, Eriksson P 2007 Changes in spontaneous behaviour and altered response to nicotine in the adult rat, after neonatal exposure to the brominated flame retardant, decabrominated diphenyl ether (PBDE 209). Neurotoxicology 28:136-142

19. Johansson N, Viberg H, Fredriksson A, Eriksson P 2008 Neonatal exposure to deca-brominated diphenyl ether (PBDE 209) causes dose-response changes in spontaneous behaviour and cholinergic susceptibility in adult mice. Neurotoxicology 29:911-919

20. Gascon M, Vrijheid M, Martínez D, Forns J, Grimalt JO, Torrent M, Sunyer J 2011 Effects of pre and postnatal exposure to low levels of polybromodiphenyl ethers on neurodevelopment and thyroid hormone levels at 4years of age. Environ Int 37:605611

21. Koh TW, Chen SC, Chang-Chien GP, Lin DY, Chen FA, Chao HR 2010 Breast-milk levels of polybrominated diphenyl ether flame retardants in relation to women's age and pre-pregnant body mass index. Int J Hyg Environ Health 213:59-65

22. Chao HA, Chen SC, Chang CM, Koh TW, Chang-Chien GP, Ouyang E, Lin SL, Shy CG, Chen FA, Chao HR 2010 Concentrations of polybrominated diphenyl ethers in breast milk correlated to maternal age, education level, and occupational exposure. J Hazard Mater 175:492-500

23. Bayley N 2005 Bayley Scales of Infants and Toddlers Development, Third edition (Bayley-III). Psychological Corporation, San Antonio

24. Anderson PJ, De Luca CR, Hutchinson E, Roberts G, Doyle LW; Victorian Infant Collaborative Group 2010 Underestimation of developmental delay by the new Bayley-III Scale. Arch Pediatr Adolesc Med 164:352-356

25. Viberg H, Mundy W, Eriksson P 2008 Neonatal exposure to decabrominated diphenyl ether (PBDE 209) results in changes in BDNF, CaMKII and GAP-43, biochemical substrates of neuronal survival, growth, and synaptogenesis. Neurotoxicology 29:152-159

26. Costa LG, Giordano G 2011 Is decabromodiphenyl ether (BDE-209) a developmental neurotoxicant? Neurotoxicology 32:9-24

27. Chen A, Dietrich KN, Huo X, Ho SM 2011 Developmental neurotoxicants in e-waste: an emerging health concern. Environ Health Perspect 119:431-438

28. Centers for Disease Control and Prevention 2010 Limitation inherent to a crosssectional assessment of blood lead levels among persons living in homes with high levels of lead in drinking water. MMWR Morb Mortal Wkly Rep 59:751

29. Birnbaum LS, Staskal DF 2004 Brominated flame retardants: cause for concern? Environ Health Perspect 112:9-17 